\title{
Modified PSO: A Bio-inspired Algorithm for Color and Gray Level Enhancement
}

\author{
Sarbjeet Singh \\ Department of Electronics and \\ Communication Engineering \\ NITTTR \\ Chandigarh, INDIA
}

\author{
Ankita Pandey \\ Department of Electronics and \\ Communication Engineering \\ NITTTR \\ Chandigarh, INDIA
}

\author{
Paramjeet Singh \\ Department of Computer \\ Science and Engineering \\ SSIET \\ Derabassi, Punjab, INDIA
}

\begin{abstract}
The use of modified version of PSO had declared the optimum solutions and act as remedy incase of particle stagnation. Traditional PSO lost its identity due to impact and strength of MPSO. Recent literatures show how modified particle swarm had achieved its name and fame over its parental algorithm called as PSO by optimizing. In this paper we exploit its advantage over image enhancement for improving image contents. This makes handy for visualizing the information from enhanced images. Work deal with use of parameterized transformation and objective function for local/global information and entropy/edge information respectively by modified PSO. Quality of Image is controlled by scaling factor and helps us in situation like gamut. Enhancement is wide study and we had attempted to use MPSO for this field. Results clearly visualize the effect of color image enhancement and gray scale level enhancement.
\end{abstract}

\section{General Terms}

segmentation; transformation function; objective function; histogram equalization.

\section{Keywords}

HIS color space; CIE; GLE; MPSO.

\section{INTRODUCTION}

The word enhancement signifies the upgrade or boosting up of respective subject. In case the subject had been the image file then it termed as image enhancement. Image enhancement is a vital technique in image processing by which clear view of needed information is brought out source image file. These enhancement methods are divided into two categories:

- Spatial Domain Method.

- Frequency Domain Method.

Whenever we are dealing with pixels of image for image enhancement then it is termed as spatial domain technique. In other word we do fourier transform of certain image is computed and then inverse fourier transform to get suited results. Such operations are dealt in order to get brightness, contrast or distribution at gray scales. In image processing, histogram transformation is regarded as fundamental process for contrast enhancement of gray level images [2]. This process is likely used for high level operation ie. Detection identification.

The word pseudo coloring plays a vital role in "color" to gray scale image based on color mapping [1]. Thus many times histogram is employed by many transformation functions to get contrast enhancement. Defining it in other words we say this process maximizes the information content of source image. Many evolutionary algorithms have been recently employed for image enhancement [3] [4] [5] [6] [19]. True image enhancement must specify clear characteristics', color sensitivity and models of color. Various studies have been done on color enhancement. Bright literature enlightens the way of using various genetic algorithms for color image enhancement and its gray scale conversion[7].

This paper involves the use of modified particle swarm optimization (MPSO) for color image enhancement and gray level image contrast enhancement. For color image enhancement, parameters are optimized by MPSO like edge and entropy. Here scaling factor $\alpha$ is calculated form intensity and color images which are enhanced. Sometime pixel come across gamut problem during scaling, this problem is solved by considering HIS color space [8]. Rest of the paper is organized as follows:

In section 2 color image and gray level enhancement and its evolution criteria is discussed. In section 3 methodology of MPSO is discussed made. At last section 4 puts us to conclusion.

\section{Color Image Enhancement \& Gray} Scale Level Enhancement (CIE \& GLE) and Evolution Criteria.

Transformation plays a vital role in image enhancement technique. Transformation takes the intensity value of every pixel form input image and liberates fresh intensity value for respective pixel. This process enhances the quality of images. To control the quality of enhanced images/evaluation of enhanced images we use evaluation function.

\subsection{Functions used for enhancement.}

Pixel by pixel manipulation is dealt by using transformation functions. This function generates new intensity value for every pixel. Let original image be $g(i, j)$ made of different gray level in range of $[0, \mathrm{~L}-1]$. Histogram of original image

$g(i, j)$ is denoted as $h(x)$ where $x$ is gray level pixel and varies from 0 to $\mathrm{L}-1$. If number of pixels at $\mathrm{g}(\mathrm{i}, \mathrm{j})$ is $\mathrm{N}$. Then histogram is evaluated as (HE);

$$
\begin{aligned}
& y=f_{H E}(x) ; \\
& =\left[H(x) \cdot \frac{(L-1)}{N}\right] ;
\end{aligned}
$$


Here $f_{H E}(x)$ is histogram function of HE.

Local enhancement method uses novel transformation on pixel considering intensity distribution among its neighborhood pixel [9]. Its main example is adaptive histogram equalization [1]. Its gives best results when dealt with medical images. Various approaches have been made by modified histogram equalization with variable enhancement [10]. Function proposed had an advantage of taking both local and global information for image enhancement. Local information is liberated from user defined window of size ( $n \quad X n)$. Transformation is defined as:

$g(i, j)=K(i, j)[f(i, j)-c \cdot m(i, j)]+m(i, j)^{a}$

Here

- $\quad \mathrm{a}, \mathrm{c}$ are parameters.

- $m(i, j)$ is local mean of input image over (n X n) window.

$m(i, j)=\frac{1}{n \times n} \sum_{x=0}^{n-1} \sum_{y=0}^{n-1} f(x, y)$

- $\mathrm{K}(\mathrm{i} \mathrm{j})$ is enhancement function take both local and global information into account.

$K(i, j)=\frac{k \cdot D}{\sigma(i, j)+b}$

(5)

$>\mathrm{k}, \mathrm{b}$ are parameters.

$>\quad \mathrm{D}$ is global mean and defined as

$$
D=\frac{1}{M X N} \sum_{i=0}^{M-1} \sum_{j=0}^{N-1} f(i, j)
$$

$>\sigma(i, j)$ is local deviation of input image over ( $\mathrm{n} \mathrm{X} \mathrm{n)} \mathrm{window}$

$$
\begin{aligned}
& \sigma(i, j)= \\
& \sqrt{\frac{1}{n X n} \sum_{x=0}^{n} \sum_{y=0}^{n}(f(x, y)-m(i, j))^{2}} \\
& \text { (7) }
\end{aligned}
$$

Thus the transformation function changes to:

$$
g(i, j)=\frac{k \cdot D}{\sigma(i, j)+b}[f(x, y)-c \cdot m(i, j)]+m(i, j)^{a}
$$

Above transformation tells us that contrast of image is being stretched considering local mean as center of stretch. Here four parameters a, b, c and $\mathrm{k}$ gives high variation in processing of image[18].

\subsection{Evaluation criteria}

In evaluation of quality of enhanced image, objective function is required. Literature speaks of many objective functions [11] [12] [13]. Objective function comprises of summation of edge intensity, value of entropy and edge pixels. Its is common sense that enhanced image shows higher intensity of edges and number of edges than original image[14]. This information is insufficient for testing purposes, so we use entropy value which gives content information of images.
When values of distribution of intensities are uniform/regular then histogram is said to be equalized. Objective function is considered as:

$F\left(I_{e}\right)=\log \left(\log \left(E\left(I_{S}\right)\right)\right) \cdot \frac{P\left(I_{s}\right)}{M X N} \cdot H\left(I_{e}\right)$

Here in this equation $I_{e}$ is enhancing image of $I_{o}$ original image as resultant from transformation equation. $E\left(I_{S}\right)$ is the sum of M X N pixel intensities of sobel edge image $I_{S}$. In this equation edges can be detected by many good detector algorithm like sobel, laplacian and canny. Entropy value can be calculated on enhanced image $I_{e}$ as:

$H\left(I_{e}\right)=-\sum_{i=0}^{255} e_{i}$

Where $e_{i}=h_{i} \log _{2}\left(h_{i}\right)$,

Here $h_{i}$ is probability of occurrence of $i^{\text {th }}$ intensity value of $I_{e}$ image.

\section{MPSO Algorithm}

\subsection{Methodology}

Particle Swarm Optimization (PSO) is population based, self adaptive and stochastic optimization technique. It was developed by Kennedy and Eberhart [15], based social behavior of collection animals. It is search technique that traces its evolution to emergent motion of group of birds searching for food. PSO has been trendy used to find optimum results in order to minimize the cost function. In multidimensional space where the optional solution is sought, each particle in swarm is moved toward optimal point by adding a velocity with its position. Velocity here is governed by set of rules that control the dynamic s of swarm. When PSO is applied one should consider limitation on representation of initial population, position, velocity and fitness strategies. Traditional PSO consist of few step

- Generation of particles

- Fitness examination

- Position and velocity update

- Termination criterion determination

Firstly intensity image is constructed from colored image with help of I (intensity) component form HIS color space [16]. Parameterized transformation function in equation (8) is used which incorporate global and local information of original image. This transformation function is applied on each pixel of original image taking parameter value from each particle, it generates a new modified intensity value for every pixel. Then quality is measured by objective function (9). Fitness value is generated by all particles is calculated, gBest and pBest is 
calculated.

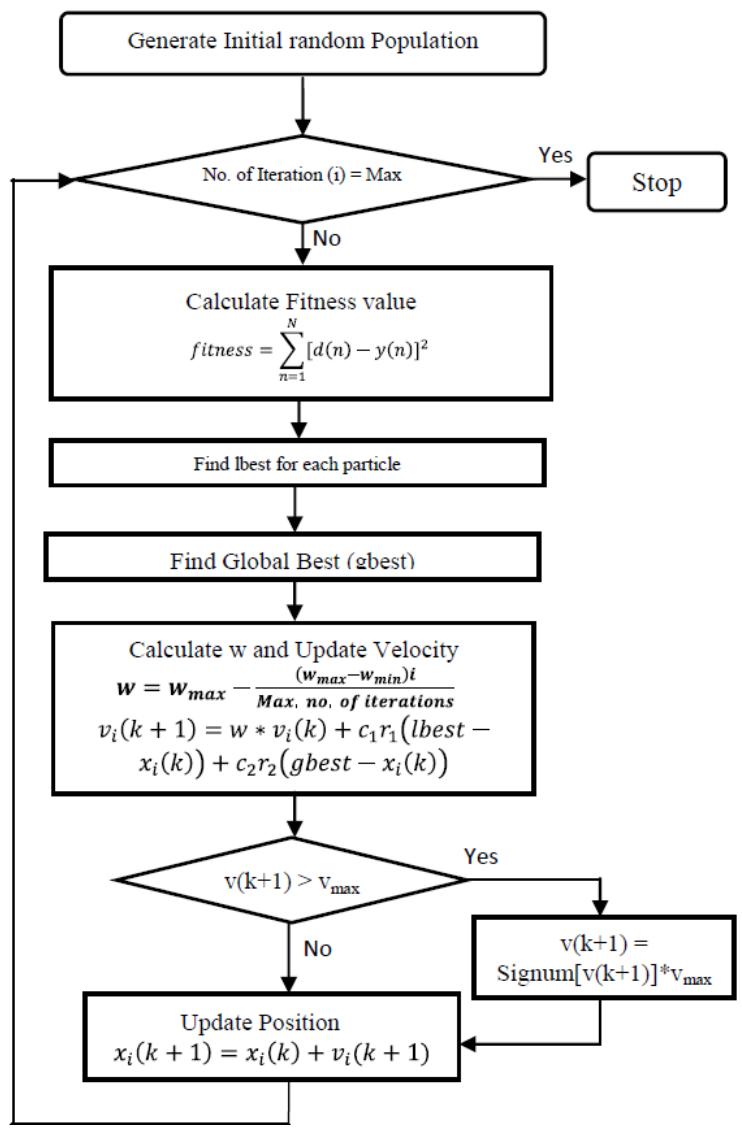

Fig-1 Modified Particle Swarm Optimization.

If $\mathrm{x}$ and $\mathrm{v}$ are particle position and velocity respectively in $\mathrm{d}$ dimensional search space then $\mathrm{i}^{\text {th }}$ particle position is represented as $x_{i}=\left(x_{i 1}, x_{i 2}, \ldots \ldots, x_{i d}\right)$ and velocity is represented as $v_{i}=\left(v_{i 1}, v_{i 2}, \ldots \ldots, v_{i d}\right)$. In MPSO earlier achieved best positions are recorded and are represented

as pBest $_{i}=$

$\left(p\right.$ Best $_{i 1}, p$ Bset $\left._{i 2}, \ldots \ldots ., p B e s t_{i d}\right)$. Enhanced image produced by gBest location is called as enhanced version of intensity version.

Particle which is best among the group is said as gBest. Updating equation of velocity and position for MPSO is given as:

$V_{i d}^{k+1}=w * V_{i d}^{k}+\alpha_{1} * R_{1} *\left(p\right.$ Best $\left._{i d}-x_{i d}^{k}\right)+$

$\alpha_{2} * R_{2} *\left(\right.$ gBest $\left._{i d}-x_{i d}^{k}\right)$

$X_{i d}^{k+1}=X_{i d}^{k}+V_{i d}^{k+1}$

Where $\mathrm{i}=1,2 \ldots \ldots N_{p}$ and

$\mathrm{d}=1,2 \ldots \ldots N_{g}$

$\mathrm{W}$ is inertial weight

$\alpha_{1}$ and $\alpha_{2}$ is acceleration constants and $R_{1}$ and $R_{2}$ is random numbers in $[0,1]$

$V_{i d}^{k}$ is current velocity

$V_{i d}^{k+1}$ is modified velocity

$X_{i d}^{k}$ is current position

$X_{i d}^{k+1}$ is modified position at iteration (K $+1)$

\section{$\alpha_{1}$ and $\alpha_{2}$ accelarationconstant}

Pulls every particle towards pBest and gBest positions. These constants are set to value 2.0 because high values results abrupt movement toward or past target region and low values results particles to move freely far from target region before being tugged back.

Suitable selection of inertial weight gives us balance between global and local exploration. It is represented as:

$W=W_{\max }-\frac{W_{\max }-W_{\min }}{\text { iter }_{\max }} *$ iter

This values of inertial weights showed great performance when they were linearly decreased, modified equation comes out to be:

$W=\left(W_{\min }-W_{\max }\right) *\left(\frac{\text { iter }_{\max }-\text { iter }}{\text { iter }_{\max }}\right)+w_{\min }$

In a color image three components $\mathrm{R}, \mathrm{G}$ and $\mathrm{B}$ are modified this results change in hue of the color vector which is needed. For preserving this hue we make each component with same scale. Scaling factor defined as ration of intensity value of original image and enhanced image. There is a case called gamut, in which RGB value does not lie in range of [0-255], this case is solved by calling HIS color Space [16]. Methodology of Modified PSO is clearly illustrated in flow chart, Figure 1[20].

\subsection{Parameter Setting}

MPSO is totally dependent on parameters like inertial weight. Max and min value of inertial weight are set as 0 and 2. It is so, process when start with maximum inertial value and gradually reduces to minimum. Thus inertial weight is big and explores large area in solution space, but inertial component become small as time passes and it exploit better results in solution space.

Parameters $\alpha_{1}, \alpha_{2}$ and $R_{1}, R_{2}$ are positive acceleration constants, a random number $[0,2]$ and $[0,1]$ respectively. Acceleration constant is fixed as 1.3. In case of equation (8), parameters a, b, c, k are chosen as $[0,1.5][0,0.5][0,1]$ $[0.5,1.5]$ respectively. Results noted that slight change in value of $b$ resulted in stretching the intensity of image in large amount [17]. So range in altered and is fixed to [1, D/2], here $\mathrm{D}$ is global mean of original image. In algorithm initial 
velocities for a, c, $\mathrm{k}$ parameters are set randomly between $[0,0.5]$ and for $b$ is kept as $[0,10]$ randomly.

TABLE I. FITTNESS TABLE AN DETAILOF ORIGNAL IMAGE

\begin{tabular}{|l|l|l|l|}
\hline TABLE HEAD & NAME OF FILE & SIZE (MXN) & $\begin{array}{l}\text { FITTNESS } \\
\text { VALUE }\end{array}$ \\
\hline 1. & $\begin{array}{l}\text { BLACK } \\
\text { MUSTANG }\end{array}$ & 272 X 265 & 15.0416 \\
\hline 2. & SIKH GREET & 515 X 344 & 10.5400 \\
\hline 3. & $\begin{array}{l}\text { WATCH } \\
\text { TOWER }\end{array}$ & 441 X 666 & 11.78793 \\
\hline 4. & SEA BEACH & 570 X 756 & 9.4075 \\
\hline
\end{tabular}

TABLE II. FITNESS TABLE FOR ENHANCED AND GRAY LEVEL IMAGE

\begin{tabular}{|l|l|l|l|}
\hline $\begin{array}{l}\text { TABLE } \\
\text { HEAD }\end{array}$ & & \multicolumn{2}{|c|}{$\begin{array}{c}\text { FITNESS :MPSO } \\
\text { ALGORITM }\end{array}$} \\
\hline & $\begin{array}{l}\text { NAME OF } \\
\text { FILE }\end{array}$ & $\begin{array}{l}\text { ENHANCED } \\
\text { IMAGE } \\
\text { FITNESS }\end{array}$ & $\begin{array}{l}\text { GRAYSCALE } \\
\text { IMAGE } \\
\text { FITNESS }\end{array}$ \\
\hline 1. & $\begin{array}{l}\text { BLACK } \\
\text { MUSTANG }\end{array}$ & 17.6728 & 1.04166 \\
\hline 2. & $\begin{array}{l}\text { SIKH } \\
\text { GREET }\end{array}$ & 18.5084 & 0.54008 \\
\hline 3. & $\begin{array}{l}\text { WATCH } \\
\text { TOWER }\end{array}$ & 15.0685 & 0.78793 \\
\hline 4. & $\begin{array}{l}\text { SEA } \\
\text { BEACH }\end{array}$ & 20.2902 & 0.40753 \\
\hline
\end{tabular}

\section{CONCLUSION}

In this paper we concluded that modified PSO is again relevant to its image processing application. Images (a) represent original image, (b) is histogram equalized image, (c) represents enhance image BY MPSO and (d) is gray scale level enhanced image by MPSO. Though both results showed better visibility details but histogram images removes the meshes visibility mustangs image but MPSO gives clear view by little darkening it. Histogram produce too much contrast and brightness in image like in case of watch tower, but PSO shows light colored image of watch tower making it feel good for eyes. Results produced shows that MPSO had great effect on images and is powerful algorithm for making colored images to gray scale images. This conversion could be helpful in coming medical researches where locating texture and finding lesion in mammographic images and CT/MRI images.

\section{References}

[1] R.C.Gonzales, R.E. woods, Digital Image Processing. Newyork: Addison-wesley, 1987

[2] R.C Gonzales, B.A.Fittes, "Grey Level transformation for interactive image enhancement," mechanism and machine theory, vol.12,pp.111-112, 1977.

[3] R. Poli, S. Cagnoni, "Evolution of pseudo-coloring algorithms for image enhancement," Univ. Birmingham, Birmingham, U.K., Tech. Rep. CSRP-1997.

[4] C. Munteanu, V. Lazarescu, "Evolutionary contrast stretching and detail enhancement of satellite images," In Proc. Mendel, Berno, Czech Rep., pp. 94-99, 1999.

[5] C. Munteanu , A. Rosa, "Evolutionary image enhancement with user behavior modeling," ACM SIGAPP Applied Computing Review, vol. 9, no. 1, pp. 814, 2001.

[6] F. Saitoh, "Image contrast enhancement using genetic algorithm," in Proc.IEEE SMC, Tokyo, Japan, pp. 899 904, 1993.

[7] G. Runqiu, L. Junfeng, L. Xiaochun, "The infrared image enhancement and the correlative technique based on the parallel genetic algorithm," XIDIAN Univ.J., vol. 31, pp. 6-8, 2004..

[8] S. K. Naik and C. A. Murthy, "Hue-preserving color image enhancement without gamut problem," IEEE Transactions on Image Processing, vol. 12, no. 12, pp. 1591-1598, 2003.

[9] C. Munteanu, A. Rosa, "Gray-scale enhancement as an automatic process driven by evolution," IEEE Transaction on Systems,Man and Cybernatics-Part B:Cybernetics, vol. 34, no. 2, pp. 1292-1298, 2004

[10] Kota murahira, takashi Kawakami, Akira Taguchi, "Modified histogram Equalization for Image enhancemnt," IEEE procedding of the $4^{\text {th }}$ ISCCSP cyprus, 2010

[11] S. Jingquan, F. Mengyin, and Z. Chanjian, "An image enhancementalgorithm based on chaotic optimization," Computer Engineering and applications, vol. 27, pp. 46, 2003 .

[12] G. Runqiu, L. Junfeng, and L. Xiaochun, "The infrared image enhancement and the correlative technique based on the parallel genetic algorithm," XIDIAN Univ.J., vol. 31, pp. 6-8, 2004.

[13] T. Xiaodong and L. Zhong, "Compare and analysis of enhancement methods of sonar image," Ship Electronics Eng.J., vol. 26, pp. 154-157, 2006.

[14] Mahdi, Mengjie Zhang, Mark Johnston, "Improving Edge Detection using Particle swarm Optimization in Noisy Images," in proceeding of $25^{\text {th }}$ ICIVC, NewZealand, IEEE Press, 2010 
[15] J. Kennedy, R. Eberhart. "Particle swarm optimization". IEEE International Conference Neural Networks, vol.4, 1995, pp.1942-1948.

[16] S. K. Naik and C. A. Murthy, "Hue-preserving color image enhancement without gamut problem," IEEE Transactions on Image Processing, vol. 12, no. 12, pp. 1591-1598, 2003.

[17] C. Munteanu and A. Rosa, "Gray-scale enhancement as an automaticprocess driven by evolution," IEEE Transactions on Systems,Man andCybernatics-Part B:Cybernetics, vol. 34, no. 2, pp. 1292-1298, 2004.

[18] Apurba Gorai, Ashish Ghosh, "Hue-Preserving Color Image Enhancement Using Particle Swarm Optimization" IEEE conference on recent advances in intelligent computational systems (RAICS), pp.563 568,2011

[19] S. K. Pal, D. Bhandari, M. K. Kundu, "Genetic algorithms for optimal image enhancement," Pattern Recognition Letter, vol. 15, pp. 261-271,1994

[20] Lipika Gupta, Rajesh Mehra, "Modified PSO based Adaptive IIR Filter Design for System Identification on FPGA", IJCA vol. 22, pp. 1-7, 2011

\section{BIBLIOGRAPHY}

ANKITA PANDEY: Ankita Pandey received her B.Tech degree from BIT's Durg, Pt. Ravi Shankar Shukla University,
Raipur, Chhatisgarh, India in 2007 and She is pursuing her M.E from NITTTR, Panjab University, Chandigarh, India. She plans to work toward Ph.D degree In Electronics and Communication. Miss. Pandey's interests are Image processing and Microcontrollers.

SARBJEET SINGH: Sarbjeet Singh was born in Punjab on $31^{\text {st }}$ Dec, 1985. He received his B.Tech degree in Electronics and Communication Engineering from Punjab Technical University, Punjab, India in 2009 and he is pursuing his M.E from NITTTR, Panjab University, Chandigarh, India. He plans to work toward Ph.D degree in Electronics. Mr. Sarbjeet Singh's interests are in Wireless Communication, Neural Network, Image processing and computation. Mr. Sarbjeet Singh is life member of ISTE.

PARAMJEET SINGH: Paramjeet Singh was born in Punjab on $21^{\text {st }}$ Oct, 1985 . He received his B.E degree in Computer Science and Engineering SLIET, Longowal, Punjab Technical University, Punjab, India in 2009 and he is pursuing his M.Tech from SSIET , Punjab Technical University, Punjab, India. He plans to work toward Ph.D degree in Computer Science and Engineering. Mr. Paramjeet Singh's interests are in Neural Network, Image and Video processing, Games Development. 

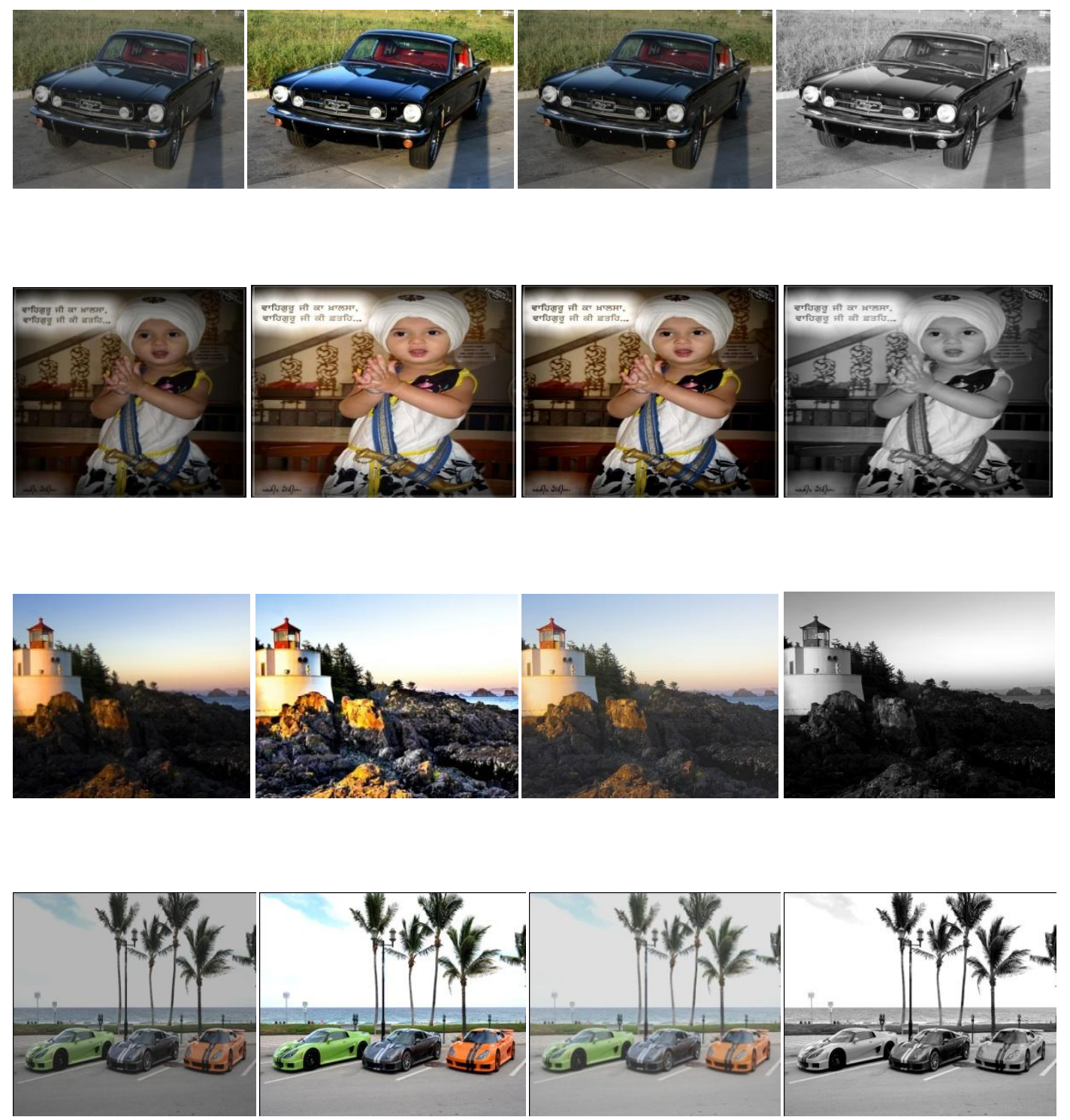

a) Original Image

b) Histogram Image

c) MPSO Image

d) Gray Scale Image 